\title{
From Turing instability to fractals
}

\author{
J. G. Huang, J. M. Christian, G. S. McDonald, J. Jahanpanah \\ Joule Physics Laboratory, School of Computing, Science and Engineering, Institute for Materials Research, \\ University of Salford, Salford M5 4WT, U.K. \\ P. Chamorro-Posada \\ Departmento de Teoría de la Señal y Comunicaciones e Ingeniería Telemática, Universidad de Valladolid, \\ ETSI Telecomunicación, Campus Miguel Delibes s/n, 47011 Valladolid, Spain
}

\begin{abstract}
A generic criterion for the generation of spontaneous fractal patterns is proposed, which has independence with respect to system nonlinearity. We also report the first transverse spatial optical fractals found in dispersive and absorptive ring cavities, and our analysis is fully confirmed by numerical simulations. (C) 2007 Optical Society of America

OCIS codes: (190.4400) Nonlinear Optics, Materials; (190.9540) Nonlinear Optics, Self-Action Effects.
\end{abstract}

\section{Introduction}

Complexity focuses on commonality across subject areas and forms a natural platform for multidisciplinary activities. Typical generic signatures of complexity include: (i) spontaneous occurrence of simple patterns (e.g. stripes, squares, hexagons) emerging as dominant nonlinear modes [1], and (ii) the formation of a highly complex pattern in the form of a fractal (with comparable levels of detail spanning decades of scale). Recently, a firm connection was established between these two signatures, and a generic mechanism was proposed for predicting the fractal generating capacity of any nonlinear system [2].

The mechanism for fractal formation is of a very general nature: any system whose Turing threshold curves exhibit a large number of comparable spatial-frequency instability minima are potentially capable of generating fractal patterns. Spontaneous spatial fractals were first reported for a very simple nonlinear system: the diffusive Kerr slice with a single feedback mirror [3]. These Kerr-slice fractals are distinct from both the transverse fractal eigenmodes of unstable-cavity lasers [4], and also from the fractals found in optical soliton-supporting systems $[5,6]$. On the one hand, unstable-cavity fractals may be regarded as a linear superposition of diffraction patterns with different scale lengths, each of which arises from successive round-trip magnifications of an initial diffractive seed. On the other hand, fractals formed in the Kerr slice result entirely from intrinsic nonlinear dynamics (i.e. light-matter coupling leading to harmonic generation and/or four-wave mixing cascades). These processes conspire to generate new spatial frequencies that, in turn, can produce optical structure on smaller and smaller scales, down to the order of the optical wavelength.

Here we report the first predictions of spontaneous fractal patterns inside driven damped ring cavities containing a thin slice of nonlinear material. Both dispersive (i.e. diffusive-relaxing Kerr [3]) and absorptive (i.e. MaxwellBloch saturable absorber [7]) are considered. New linear analyses have shown that the transverse instability spectra of these two cavity systems possess the requisite comparable minima that predict the capacity for the spontaneous generation of fractal patterns. Extensive numerical simulations, in both one and two transverse dimensions, have verified that both the dispersive and absorptive cavities do indeed give rise to nonlinear optical fractals in the transverse plane. Our results confirm that the mechanism for fractal formation has independence with respect to the details of the nonlinearity.

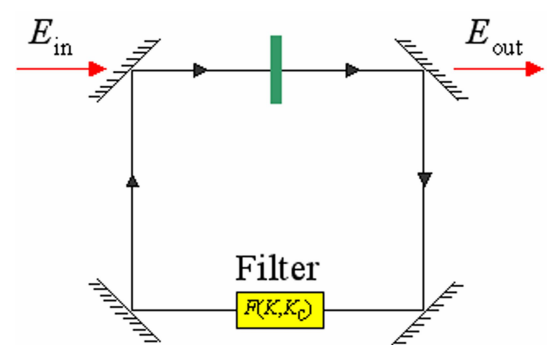

(a)

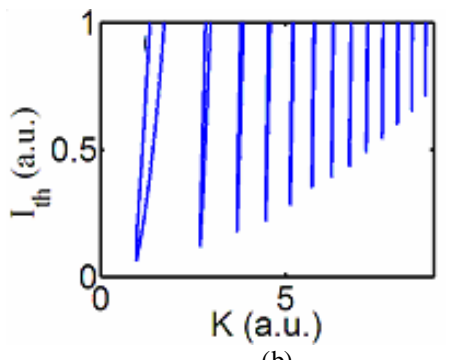

(b)

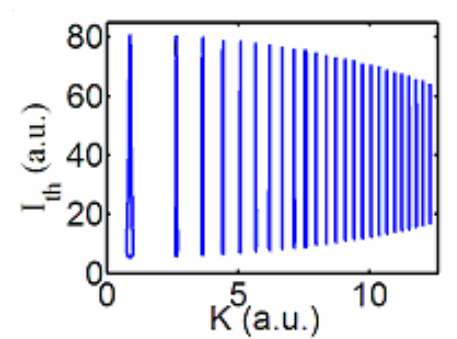

(c)

Fig. 1. (a) Schematic diagram of a ring cavity with a thin slice of nonlinear medium and a spatial filter $F\left(K^{2}, K_{C}^{2}\right)$. Typical Turing instability threshold curves for (b) diffusive-relaxing Kerr, and (b) Maxwell-Bloch saturable absorber cavities. 


\section{Optical feedback \& cavity boundary condition}

An essential ingredient for the generation of fractals is the presence of a feedback mechanism [2]. Feedback drives the cascade process that is responsible for the creation of higher spatial wavenumbers, and which ultimately leads to the "structure across decades of scale" character of the fractal pattern. Cavity geometries [see Fig. 1(a)] are therefore ideal candidates as potential optical fractal generators. In the free-space path, the electric field undergoes Helmholtz diffraction so that in Fourier space, the ring cavity boundary condition can be written as

$$
E(\mathbf{K}, 0, t)=a \delta(\mathbf{K})+\sqrt{R} \exp \left(i \phi_{0}\right) F\left(K^{2}, K_{C}^{2}\right) \exp \left[-i \theta\left(K^{2}\right)\right] E\left(\mathbf{K}, l, t-t_{R}\right)
$$

where $F\left(K^{2}, K_{C}^{2}\right)$ is a spatial filter (parameterized by a cut-off wavenumber $K_{C}$ ) that controls the maximum allowed transverse wavenumber in the cavity, and $\theta\left(K^{2}\right)=\sigma 2 K^{2} /\left[1+\left(1-K^{2} / k_{0}^{2}\right)^{1 / 2}\right]$ is the Helmholtz diffraction operator. The out-coupling mirror has an intensity reflectivity $R, t_{R}$ is the cavity transit time, $\varphi_{0}$ is the linear mistuning, and the slice has a (negligible) thickness $l$. Also, $\sigma \equiv L / 2 k_{0}, k_{0}$ is the free space longitudinal wave number, $a$ is the amplitude of the plane-wave pump field, and $\delta(\mathbf{K})$ is the Dirac delta function. By setting $k_{0} \rightarrow \infty$, the paraxial propagation factor $\theta\left(K^{2}\right) \sim \sigma K^{2}$ can be recovered, and when the spatial filter is removed (i.e. by setting $F=1$ ), the paraxial cavity boundary condition is obtained.

\section{Dispersive nonlinearity}

The simplest dispersive nonlinearity is provided by the relaxing-diffusing Kerr effect. The field $E$ inside the slice and the photoexcitation density $n$ are governed by

$$
\frac{\partial E}{\partial z}=i \chi n E \quad \text { and } \quad \tau \frac{\partial n}{\partial t}-l_{D}^{2} \nabla_{\perp}^{2} n+n=|E|^{2}
$$

respectively. $\chi$ parameterizes the Kerr nonlinearity (positive for self-focusing, negative for self-defocusing), and the coefficients $\tau$ and $l_{D}$ are the relaxation time and diffusion length, respectively, of $n$. Equations (2) are supplemented by the boundary condition (1). A linear stability analysis [2] of this system shows that the threshold condition for Turing instability is

$$
1+l_{D}^{2} K^{2}+2 \sqrt{R} \chi l I_{t h} \frac{\sin \left(\phi_{0}+\chi l I_{t h}-\sigma K^{2}\right)}{1+R-2 \sqrt{R} \cos \left(\phi_{0}+\chi l I_{t h}-\sigma K^{2}\right)}=0 .
$$

The threshold curves [see Fig. 1(b)] possess the qualitative features necessary for the generation of spontaneous fractal patterns: successive and comparable spatial frequency minima.

Rigorous simulations have shown that the Kerr cavity is indeed capable of generating fractal patterns. In a single$K$ configuration, where the filter attenuates all those spatial wavenumbers outside the first instability band, it is found that simple stripe patterns emerge. Once this stationary pattern has been reached, the filter is removed to allow all waves to propagate. Energy is transferred to higher spatial frequencies, and the simple strip pattern acquires successive level of fine detail at a rate that depends upon the system parameters. By analysing the power spectrum $P(K)$ it can be seen that a fractal pattern emerges relatively rapidly (see Fig. 2). Eventually, the system enters a dynamic equilibrium (within typically less than a hundred transits) where the average power spectrum remains unchanged even though the pattern continues to evolve in real space. When this statistically invariant state
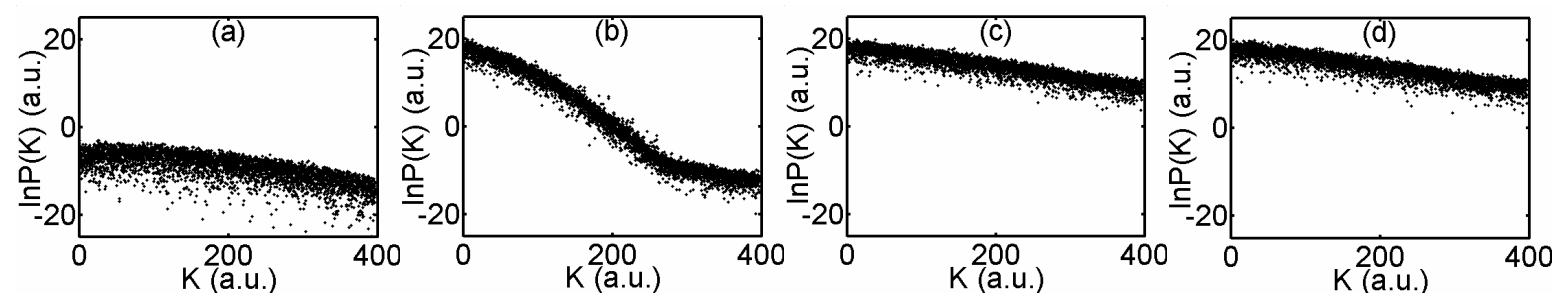

Fig. 2. Typical power spectrum evolution in time: (a) $t=t_{R}$, (b) $t=25 t_{R}$, (c) $t=100 t_{R}$, (d) $t=2500 t_{R}\left(\tau=0, l_{D}=0.4\right.$ and $\left.a=2\right)$. 
is attained [see Fig. 2(c) and 2(d)], the pattern is referred to as a scale-dependent fractal. An appreciable portion of the dynamic state is well described by a linear relationship $\ln P(K)=a+b K$, where $a$ and $b$ are constants, and this type of behaviour is one of the characteristics of a fractal pattern [2].

\section{Absorptive nonlinearity}

We have recently found that a thin-slice Maxwell-Bloch saturable absorber [7], can also generate fractal patterns. After adiabatic elimination, the model equations for the electric field $E$ and population inversion $w$ are

$$
\frac{\partial E}{\partial z}=\frac{\alpha_{0}}{2} \frac{1-i \Delta}{1+\Delta^{2}} E w \quad \text { and } \quad \partial_{t} w-l_{D}^{2} \nabla_{\perp}^{2} w+\frac{1+w}{T_{1}}=-\frac{T_{2}}{1+\Delta^{2}}|E|^{2} w
$$

respectively. $T_{1}$ and $T_{2}$ are the decay times for the population inversion and polarization, respectively, $\Delta$ is the medium detuning parameter (a measure of the difference between pump and atomic-resonance frequencies) and $\alpha_{0}$ is the absorption coefficient. Depending upon $|\Delta|$, this system can be either purely absorptive $(\Delta=0)$ or purely dispersive, $(|\Delta|>>1$ ). A linear analysis of Eqs. (4), together with a generalized boundary condition (which allows for attenuation), yields the threshold condition for Turing instability,

$$
\frac{1+I_{t h}}{T_{1}}+l_{D}^{2} K^{2}=\frac{I_{t h} \alpha l \bar{R}}{\left(1+I_{t h}\right) T_{1}} \frac{\cos \Gamma_{t h}+\Delta \sin \Gamma_{t h}-\bar{R}}{1+\bar{R}^{2}-2 \bar{R} \cos \Gamma_{t h}}
$$

where

$$
\Gamma_{t h} \equiv \phi_{0}+\frac{\Phi}{1+I_{t h}}-\sigma K^{2}, \quad \bar{R}\left(I_{t h}\right) \equiv \sqrt{R} \exp \left(-\frac{\alpha l}{2\left(1+I_{t h}\right)}\right),
$$

$\Phi=1 / 2 \alpha L \Delta$ and $\alpha=\alpha_{0} /\left(1+\Delta^{2}\right)$. For this system, one finds that the threshold spectrum comprises a series of adjacent instability islands [see Fig. 1(c)]. Simulations have revealed that the Maxwell-Bloch system (4) can also support fractals. The single- $K$ patterns turn out to be hexagonal arrays [see Fig. 3(a)], familiar from conventional pattern formation [1,3]. Once this state has been reached, the spatial filter is removed and one can observe a rapid transition toward a fractal pattern [Figs. 3(b-d)]. The qualitative behaviour of fractals patterns in both dispersive and absorptive systems are found to be the same, confirming the assertion of independence with respect to nonlinearity.

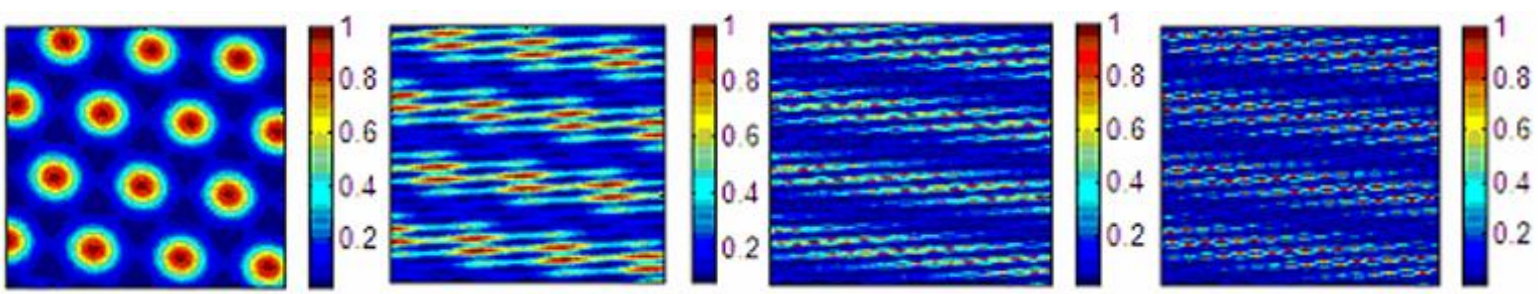

Fig. 3. Transverse intensity distribution showing the transition from a conventional (single- $K$ ) pattern - a hexagonal array - to a fractal mode in a thin-slice Maxwell-Bloch ring cavity. Self-similarity persists down to spatial scales of the order of the optical wavelength.

\section{References}

[1] J. B. Geddes et al., "Hexagons and squares in a passive nonlinear optical system," Phys. Rev. A 5, 3471-3485 (1994).

[2] J. G. Huang and G. S. McDonald, “Spontaneous optical fractal pattern formation,” Phys. Rev. Lett. 94, 174101 (2005).

[3] G. D’Alessandro and W. J. Firth, “Hexagonal spatial patterns for a Kerr slice with a feedback mirror,” Phys. Rev. A 46, 537-548 (1992).

[4] J. G. Huang et al., "Fresnel diffraction and fractal patterns from polygonal apertures,” J. Opt. Soc. Am. A 23, 2768-2774 (2006).

[5] M. Soljacic and M. Segev, "Self-similarity and fractals in soliton-supporting systems," Phys. Rev. E 61, R1048-R1051 (2000);

[6] S. Sears et al., "Cantor set fractals from solitons," Phys. Rev. Lett. 84, 1902-1905 (2000);

[7] A. S. Patrascu et al., "Multi-conical instability in the passive ring cavity: linear analysis," Opt. Commun. 91, 433-443 (1992). 